\title{
Treatment of Metformin Decreases the Risk of Varicose Vein: A Mendelian Randomization Study
}

\section{Qinchang Chen}

Sun Yat-sen University First Affiliated Hospital

Lingling Li

Sun Yat-sen University First Affiliated Hospital

Ridong Wu

Sun Yat-sen University First Affiliated Hospital

Shenming Wang

Sun Yat-sen University First Affiliated Hospital

Chen Yao ( $\nabla$ yaochen@mail.sysu.edu.cn)

Sun Yat-Sen University https://orcid.org/0000-0003-0327-9599

\section{Research article}

Keywords: Varicose vein, Metformin, Mendelian randomization, Causal association

Posted Date: September 15th, 2020

DOI: https://doi.org/10.21203/rs.3.rs-71387/v1

License: @ (i) This work is licensed under a Creative Commons Attribution 4.0 International License. Read Full License 


\section{Abstract}

Background Varicose vein is a common illness of the vascular system which affects life quality and social function of patients. We aimed to assess the causality between metformin and varicose vein using a two-sample Mendelian randomization囚MR囚analysis based on genome-wide association study (GWAS) summary data.

Methods Twenty-five single nucleotide polymorphisms (SNPs) were selected from the GWAS summary data from Neale Lab and MRC-IEU Consortium available on the MR-base platform. Inverse variance weighted (IVW), MR-egger method, weighted median method and weighted mode were adopted. Results were evaluated by pleiotropy test using an Egger regression method and sensitivity analysis preforming a leaving-one-out (LOO) method. Analyses were performed using R package "TwoSampleMR".

Results The result of IVW method showed that one SD increased treatment of metformin was linked with approximately $10 \%$ lower risk of varicose vein $(\mathrm{OR}, 0.90 ; 95 \% \mathrm{Cl}, 0.85-0.96 ; \mathrm{P}, 6.99 \mathrm{e}-04)$. It was similar to that measured by other methods in the aspect of effect size and direction. There is no evidence to supporting genetic pleiotropy in the MR-Egger regression method (intercept $=2.5 \mathrm{e}-04, \mathrm{P}=0.33$ ). No single SNP was detected to be strongly driving the overall causal effect in a LOO sensitivity analysis. The genetically predicted treatment of metformin was negatively casually associated with varicose veins.

Conclusions This study suggested that treatment of metformin was a casual protective factor of varicose vein. Further researches are required to confirm our findings and explore the potential mechanisms of metformin on varicose vein.

\section{Introduction}

As one of the most illnesses of the vascular system, varicose vein is a syndrome of superficial venous hypertension and varices due to venous blood reflux, mainly caused by increased venous pressure, weak vein wall and insufficiency venous valves, usually occurs in the lower extremities [1-2]. Varicose vein is characterized by pigment, desquamation, itching and edema of ankle. If untreated, it will lead to venous ulceration and venous thrombosis, which seriously affects life quality of patients as well as aggravates heavy society and economic burdens [3]. Advanced age, female and obesity are important risk factors for varicose vein [4-6]. Nowadays, instead of effective medical therapy, minimally surgery is the most widely used method in clinical practice, but leaves longterm problems of reopening [3]. In the molecular level, disturbances of transforming growth factor-beta one (TGF- $\beta 1$ ) signaling pathway may contribute to varicose vein [7]. Therefore, researches are still required to improve the treatment on varicose vein.

Metformin is a classic oral anti-diabetic drug which has been clinically used to treat the patient with type 2 diabetes for over 50 years [8]. Recently, in addition to the glucose-lowering effect, some studies indicate its all-round beneficial impact including diabetes prevention, obese treatment, polycystic ovary disease treatment, anticancer effect, antiaging effect, cardioprotective effect and so on [9-11]. There are reports that the mechanism of metformin action might be significantly lessening the level of TGF- $\beta 1$ expression through AMPK activation [12-14]. There was a retrospective study published in 2019 showed that the use of metformin decreased the incidence of varicose vein in people with type 2 diabetes [15]. However, observational studies are easily affected by confounding factors and reverse causality, so that it is difficult to confirm whether there is causal association between metformin and varicose vein [16].

Mendelian randomization (MR) is a new method which considers genetic variants as instrumental variables for exposures in order to evaluate whether there are causal associations between exposures and disease outcomes [17]. Since genetic variants are randomly distributed in the population and preserve stability over time, MR is highly similar to the Randomized Controlled Trials (RCTs) which escape from confounding factors and reverse causality [18]. Moreover, it has the advantage of cost and time saving when compared with RCTs. In recent years, the rapid development of Genome-wide association study (GWAS) supplies great convenience to MR, making GWAS-based two-sample MR an efficient and feasible approach [19-20].

In this study,we intended to investigate a potentially causal association between metformin and varicose vein using MR and estimate the range of effects.

\section{Methods Study design}

This study applied two-sample MR analysis to estimate the causal association between metformin and varicose vein. The institutional review board approved the MR study due to the data from summary GWAS study and without clinical intervention to the patients. The two-sample MR study, which allows the association data of genetic variants - exposures and genetic variants - disease outcomes to come from two independent samples of the same population, greatly improving the efficiency and feasibility of etiology researches, has been widely used to identify causal associations between exposures and disease outcomes [21]. It is based on three hypotheses: (1) genetic variants is robustly related to exposures; (2) genetic variants is independent of confounding factors; (3) genetic variants affects disease outcome only through exposure factors [22]. In the study, several single nucleotide polymorphisms (SNPs) were selected as instrumental variables from the GWAS of metformin and varicose vein obtained through MR-base platform. The F statistics and Q statistics were respectively calculated to ensure small bias caused by weak instruments and low heterogeneity. Four methods of Mendelian randomization, including inverse variance weighted (IVW), weighted median method, weighted mode and MR-egger method were adopted to assess the causal association between metformin and varicose vein [23-24]. Eventually, results were evaluated by pleiotropy test using an Egger regression method and sensitivity analysis preforming a leaving-one-out method.

\section{Sources of data}

We mainly used the GWAS summary data on metformin from Neale Lab Consortium in 2017 that was included in UK Biobank(http://www.nealelab.is/blog/2017/9/11/details-and-considerations-of-the-uk-biobank-gwas). The study included a total of 8,392 cases and 328,767

Page 2/8 
controls, all of whom were Europeans. The GWAS summary data on varicose vein was developed by MRC-IEU consortium in 2018 which with 18,818 cases and 444,115 controls rooted Europe (Table 1). The above GWAS summary data was available on the MR-base platform (http://www.mrbase.org). The MRbase platform was a database which integrates GWAS data with software that automates 2-sample MR, making MR analysis more rigorous and convenient [25].

Table 1

Details of studies and datasets used for MR analysis

\begin{tabular}{|c|c|c|c|c|c|c|c|c|}
\hline $\begin{array}{l}\text { Exposure/ } \\
\text { outcomes }\end{array}$ & ID & Data source & Category & $\begin{array}{l}\text { Sample size (No. cases/ } \\
\text { No. controls) }\end{array}$ & $\begin{array}{l}\text { First } \\
\text { author }\end{array}$ & Consortium & Year & Popı \\
\hline Metformin & $\begin{array}{l}\text { UKB- } \\
\text { a:159 }\end{array}$ & $\begin{array}{l}\text { http://www.nealelab.is/blog/2017/9/11details- } \\
\text { and-considerations-of-the-uk-biobank-gwas }\end{array}$ & NA & $337159(8392 / 328767)$ & Neale & Neale Lab & 2017 & Euro \\
\hline $\begin{array}{l}\text { Varicose } \\
\text { vein }\end{array}$ & $\begin{array}{l}\text { UKB- } \\
\mathrm{b}: 15592\end{array}$ & $\begin{array}{l}\text { http://www.bristol.ac.uk/integrative- } \\
\text { epidemiology/ }\end{array}$ & Binary & 462933(18818/444115) & $\begin{array}{l}\text { Ben } \\
\text { Elsworth }\end{array}$ & MRC-IEU & 2018 & Euro \\
\hline
\end{tabular}

\section{Screening for SNPs associated with metformin as instrumental variables}

In this study, SNPs selected should be closely associated with metformin at a GWAS threshold of statistical significance as well as independent from each other to protect against bias caused by strong linkage disequilibrium (LD). It was available for us to obtain independent SNPs associated with metformin automatically from GWAS by setting p-values and linkage disequilibrium LD coefficients in the MR-Base platform. Thus, we set the $p$-value $<5 \times 10^{-8}$, linkage disequilibrium $r^{2}<0.1$ to screen SNPs significantly associated with metformin as instrumental variables according to the previous literature [26]. We identified 26 SNPs (rs10001190, rs10196106, rs10965246, rs11257655, rs115576427, rs11602873,rs11658063, rs12889801, rs13266634,

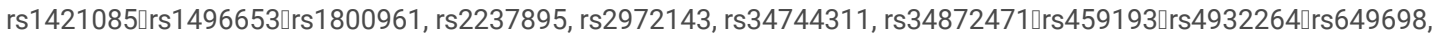

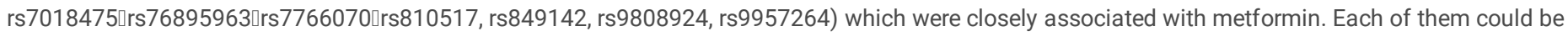
retrieved in the GWAS summary data on varicose vein authored by Ben Elsworth et al. (http://www.bristol.ac.uk/integrative-epidemiology/) [27]. One SNP (rs649698) was gotten rid of MR analysis for strand ambiguity. The rest 25 SNPs explained $0.49 \%$ of the variations in using of metformin across individuals. The F statistic calculated was more than 10 , which was generally believed to reduce bias caused by weak instruments [28]. The heterogeneity test indicated no heterogeneity on causal effect of metformin on risk of varicose vein among each of the 25 SNPs (Q statistic, 30.16; P, 0.18). Finally, 25 independent SNPs associated with metformin were included as instrumental variables in the subsequent MR analysis. The information collected included the coding numbers, effect allele(EA), non-effect allele(NEA), effect allele frequency (EAF), effect sizes ( $\beta$ ), standard errors (SE) and P values of 25 SNPs (Supplement Table 1).

\section{Statistical analyses}

In this study, screening out for independent SNPs was completed in MR-base platform, while two-sample MR analysis was preformed to assess potential associations between metformin and varicose vein using R (version 3.4.2) package "TwoSampleMR" (version 0.3.4) to achieve automated operations [29]. Four Mendelian randomization method incorporating IVW approach, weighted median method, weighted mode, and MR-Egger method were resorted to explore causal effects of metformin for varicose veins. Results of MR of were expressed as odds ratios (OR) and confidence interval (Cl). Forest plot and scatter plot showed the results more intuitively conducted by R. Differences were considered to be statistically significant if $P$-value $<0.05$.

We implemented a pleiotropy test preforming Egger regression method to test whether there was a violation of hypothesis of MR. Sensitivity analysis was adopted using a leave one out (LOO) method in which one SNP was removed in turn to estimate causal effects of the rest by IVW method for assess whether individual SNP affected MR estimates.

\section{Results}

The MR estimates of the four methods including the IVW approach, weighted median method, weighted mode, and MR-Egger method were shown as OR and $95 \% \mathrm{Cl}$ in the Table 2. The result of IVW method showed that treatment of metformin was associated with approximately $10 \%$ lower risk of varicose vein (OR,0.90; 95\% Cl, 0.85-0.96; P, 6.99e-04). The individual causal effect estimated by each SNP and the overall MR estimates including all 25 SNPs using four different methods as well as their $95 \% \mathrm{Cl}$ were demonstrated in Fig. 1. Causal estimates of two SNPs (rs34872471 and rs76895963) showed clear negative correlations between treatment of metformin and risk of varicose veins. Figure 2 revealed the regression curve of causal effect between using of metformin and risk of varicose vein. The casual association estimated by MR-Egger method, weighted median method and weighted mode method were consistent to that by IVW method, no matter in the aspect of effect size or direction (Table 2, Fig. 1 and Fig. 2).

Table 2

Mendelian randomization estimates of the associations between metformin and risk of varicose veins.

\begin{tabular}{|c|c|c|c|c|c|c|c|c|}
\hline Outcome & IVW method & & MR-Egger & & Weight median & & Weighted mode & \\
\hline \multirow[t]{2}{*}{ Varicose veins } & $\mathrm{OR}(95 \% \mathrm{Cl})$ & $P$ value & $\mathrm{OR}(95 \% \mathrm{Cl})$ & $P$ value & $\mathrm{OR}(95 \% \mathrm{Cl})$ & $P$ value & $\mathrm{OR}(95 \% \mathrm{Cl})$ & $P$ value \\
\hline & $0.90(0.85-0.96)$ & $6.99 \mathrm{e}-04$ & $0.85(0.74-0.97)$ & 0.03 & $0.90(0.83-0.97)$ & $9.10 \mathrm{e}-03$ & $0.90(0.83-0.99)$ & 0.03 \\
\hline
\end{tabular}


In this study, there is no evidence to supporting directional horizontal pleiotropy in the MR-Egger regression method (intercept $=2.5 \mathrm{e}-04, \mathrm{P}=0.33$ ). No single SNP was detected to be strongly driving the all causal effect of metformin on risk of varicose vein in a LOO sensitivity analysis. The more detail information about LOO sensitivity analysis was showed in the Fig. 3 and appendix Table 2.

\section{Discussion}

Varicose vein is a common clinical illness that limits physical and social activities of patients. Stretch socks, harden agent and surgery are the main prevention and treatment measures instead of effective medical therapy at present, but leaves the problem of long-term reopening and surgery complications [3]. Metformin is an inexpensive classical but all-round beneficial drug. In this MR study, the major finding was that metformin was casually associated with varicose vein. Our results indicated that the treatment of metformin predicted a reduction in the risk of varicose vein by $10 \%$.

Little researches were done to seek the association between treatment of metformin and risk of varicose vein. A cohort study exhibited that metformin decreased the incidence of varicose vein in type 2 diabetes patients, which was in accordance with the result in our analysis [15]. However, the truth of causal association between metformin and varicose vein without diabetes remained unknown. Concerning the unethical approaches of taking metformin in patients without diabetes, RCT is not appropriate for this issue. Mendelian randomization analysis is a new method widely used to identify causal associations in a lot of etiology researches [17]. Due to the strength of MR, this analysis is able to overcome the limitation of methodology in retrospective study, such as selection bias, indication confounder and so on. Through MR analysis, our study revealed that treatment of metformin was linked with approximately $10 \%$ lower risk of varicose veins.

The possible mechanisms of the sheltering effect of metformin against varicose veins have not been clarified. Impaired microvascular endothelial function plays an important role in generation and progress of varicose veins [30]. The sensation of venous hypertension and valvular incompetence lead to the recruitment of inflammatory cells and the release of inflammatory mediators, which was triggered by the activation of endothelial cells. It has been well demonstrated that metformin can improve the endothelial function and relieve the inflammatory reaction [31-33]. In the molecular level, a research about vascular wall pathology showed TGF- $\beta 1$ signaling pathway worked in development of varicose veins [7]. It is widely accepted that the mechanism of metformin action might be significantly lowering down the level of TGF- $\beta 1$ expression through AMPK activation [12-14]. Thus, the protection function from metformin on varicose vein might be associated with impaired endothelial function, inflammation and valvular incompetence while further investigations were required.

This study had several great advantages. First, this was the first MR study to investigate the casual relationship between metformin and varicose vein. Compared with RCT, it was an enormous saving of time, money and effort using MR method. Second, MR analysis escaped from the impact of reverse causation and confounding factors, which were common in conventional researches. Finally, the large sample size (over 750000) and robust instrumental variables (F statistics > 10) leaded to more credible causal estimates. However, our study also leaved much to be desired. First, two large consortiums included in this study were both European populations. It was questionable whether our findings could apply to other populations, such as Asian race. Second, due to the limitation from the GWAS summary data, we are not able to obtain the data in the individual level, for which our result provided a linear relationship between metformin and varicose vein while the relationship might be $U$ shape in real world. The taking dosage and frequency of metformin for individual patient were not able to assess and thus subgroup analysis were difficult to conduct. Third, through the method of MR, we were not able to investigate the possible mechanism metformin and varicose vein.

\section{Conclusion}

In this study, we found the negatively casual association between metformin and risk of varicose vein using two-sample Mendelian randomization method based on GWAS summary data. But further researches are demanded to add to the findings and explore the potential mechanism of metformin on varicose vein.

\section{Declarations}

\section{ETHICS APPROVAL AND CONSENT TO PARTICIPATE}

Not applicable.

\section{COMPETING INTERESTS}

The authors have no conflict of interests.

\section{Acknowledgements}

Not applicable.

\section{Source of Funding}

This work was supported by National Natural Science Funds for Young Scholars of China (81800416) and Guangzhou Major program of the IndustryUniversity-Research collaborative innovation (201508020116).

\section{Author Contributions}


Conception and design: QC, LL, SW, CY

Analysis and interpretation: QC, LL, RS, RW, CY

Data collection: QC, LL

Writing the article: QC, LL, RS, RW

Critical revision of the article: QC, LL, SW, CY

Final approval of the article: QC, LL, RS, RW, SW, CY

Statistical analysis: QC, LL

Obtained funding: RW, $\mathrm{CY}$

Overall responsibility: SW CY

\section{References}

1. van der Velden SK, Pichot O, van den Bos RR, Nijsten TE, De Maeseneer MG. Management strategies for patients with varicose veins (C2-C6): Results of a worldwide survey. Eur J Vasc Endovasc Surg 2015; 49: 213-20.

2. Brittenden J, Cooper D, Dimitrova M, Scotland G, Cotton SC, Elders A, et al. Five-year outcomes of a randomized trial of treatments for varicose veins. N Engl J Med 2019; 381: 912-22.

3. Jacobs BN, Andraska EA, Obi AT, Wakefield TW. Pathophysiology of varicose veins. J Vasc Surg Venous Lymphat Disord 2017; 5: 460-7.

4. Sivakumar S, Banupriya K. A cross-sectional descriptive clinical study of dermatological manifestations in obesity. Int J Res $2017 ; 3$ : 337.

5. Zolotukhin IA, Seliverstov El, Shevtsov YN, Avakiants IP, Nikishkov AS, Tatarintsev AM, Kirienko Al. Prevalence and risk factors for chronic venous disease in the general Russian population. Eur J Vasc Endovasc Surg 2017; 54: 752-8.

6. Davies H O B, Popplewell M, Singhal R, Smith N, Bradbury AW. Obesity and lower limb venous disease-The epidemic of phlebesity. Phlebology 2017;32: 227-33.

7. Serralheiro P, Soares A, Costa Almeida CM, Verde I. TGF- $\beta 1$ in vascular wall pathology: unraveling chronic venous insufficiency pathophysiology. Int J Mol Sci 2017;18: 2534.

8. An H, He L. Current understanding of metformin effect on the control of hyperglycemia in diabetes. J Endocrinol 2016; 228 : R97.

9. Aroda VR, Knowler WC, Crandall JP, Perreault L, Edelstein SL, Jeffries SL, et al. Metformin for diabetes prevention: Insights gained from the diabetes prevention program/diabetes prevention program outcomes study. Diabetologia 2017;60: 1601-11.

10. Griffin S J, Leaver J K, Irving G J. Impact of metformin on cardiovascular disease: a meta-analysis of randomised trials among people with type 2 diabetes. Diabetologia 2017; 60: 1620-9.

11. Heckman-Stoddard BM, DeCensi A, Sahasrabuddhe VV, Ford LG. Repurposing metformin for the prevention of cancer and cancer recurrence. Diabetologia 2017; 60: 1639-47.

12. Chiang CF, Chao TT, Su YF, Hsu CC, Chien CY, Chiu KC, et al. Metformin-treated cancer cells modulate macrophage polarization through AMPK-NF-kappaB signaling. Oncotarget 2017; 8:20706.

13. Feng Y, Wang S, Zhang Y, Xiao H. Metformin attenuates renal fibrosis in both AMPKalpha2-dependent and independent manners. Clin Exp Pharmacol Physiol 2017; 44: 648-55.

14. Luo T, Nocon A, Fry J, Sherban A, Rui X, Jiang B, et al. AMPK Activation by Metformin Suppresses Abnormal Extracellular Matrix Remodeling in Adipose Tissue and Ameliorates Insulin Resistance in Obesity. Diabetes 2016; 65: 2295-310.

15. Tseng CH. Metformin reduces risk of varicose veins in patients with type 2 diabetes. Diabetes Metab Res Rev 2019 ; e3206.

16. Boyko EJ. Observational research-opportunities and limitations. J Diabetes Complications 2013; 27: 642-8.

17. Emdin C A, Khera AV, Kathiresan S. Mendelian randomization. JAMA 2017; 318: 1925-6.

18. Sekula P, Fabiola Del Greco M, Pattaro C, Köttgen A. Mendelian randomization as an approach to assess causality using observational data. J Am Soc Nephrol 2016; 27: 3253-65.

19. Swerdlow DI, Kuchenbaecker KB, Shah S, Sofat R, Holmes MV, White J, et al. Selecting instruments for Mendelian randomization in the wake of genomewide association studies. Int J Epidemiol 2016; 45: 1600-16.

20. Hartwig F P, Davies N M, Hemani G, Davey Smith G. Two-sample Mendelian randomization: avoiding the downsides of a powerful, widely applicable but potentially fallible technique. Int J Epidemiol 2016; 45:1717-26.

21. Pierce B L, Burgess S. Efficient design for Mendelian randomization studies: subsample and 2-sample instrumental variable estimators. Am J Epidemiol 2013;178: 1177-84.

22. Davey Smith G, Hemani G. Mendelian randomization: genetic anchors for causal inference in epidemiological studies. Hum Mol Genet 2014; $23:$ R89-98.

23. Bowden J, Davey Smith G, Haycock P C, Burgess S. Consistent estimation in Mendelian randomization with some invalid instruments using a weighted median estimator[J]. Genet epidemiol 2016; 40: 304-14. 
24. Bowden J, Del Greco M F, Minelli C, Davey Smith G, Sheehan N, Thompson J. A framework for the investigation of pleiotropy in two-sample summary data Mendelian randomization. Stat Med 2017;36: 1783-802.

25. Hemani G, Zheng J, Elsworth B, Wade KH, Haberland V, Baird D, et al. The MR-Base platform supports systematic causal inference across the human phenome. Elife 2018; 7: e34408.

26. Schaid D J, Chen W, Larson N B. From genome-wide associations to candidate causal variants by statistical fine-mapping. Nat Rev Genet 2018; 19: 491504.

27. Elsworth B, Mitchell R, Raistrick C A, Paternoster L, Hemani G, Gaunt T. MRC IEU UK Biobank GWAS pipeline version 1. University of Bristol, 2017.

28. Burgess S, Thompson SG. Bias in causal estimates from Mendelian randomization studies with weak instruments. Stat Med 2011; 30: 1312-23.

29. Yavorska OO, Burgess S. Mendelian Randomization: an R package for performing Mendelian randomization analyses using summarized data. Int $J$ Epidemiol 2017; 46: 1734-9.

30. Castro-Ferreira R, Cardoso R, Leite-Moreira A, Mansilha A. The role of endothelial dysfunction and inflammation in chronic venous disease. Ann Vasc Surg 2018; 46: 380-93.

31. Hung $\mathrm{CH}$, Chan S H, Chu P M, Lin HC, Tsai KL. Metformin regulates oxLDL-facilitated endothelial dysfunction by modulation of SIRT1 through repressing LOX-1-modulated oxidative signaling. Oncotarget 2016;7: 10773.

32. Sambe T, Mason R P, Dawoud H, Bhatt DL, Malinski T. Metformin treatment decreases nitroxidative stress, restores nitric oxide bioavailability and endothelial function beyond glucose control. Biomed Pharmacother 2018; 98: 149-56.

33. Ghosh S, Lakshmanan A P, Hwang M J, Kubba H, Mushannen A, Triggle CR, Ding H. Metformin improves endothelial function in aortic tissue and microvascular endothelial cells subjected to diabetic hyperglycaemic conditions. Biochem Pharmacol 2015; 98: 412-21.

34. Segiet OA, Brzozowa-Zasada M, Piecuch A, Dudek D, Reichman-Warmusz E, Wojnicz R. Biomolecular mechanisms in varicose veins development. Ann Vasc Surg 2015; 29: 377-84.

\section{Figures}

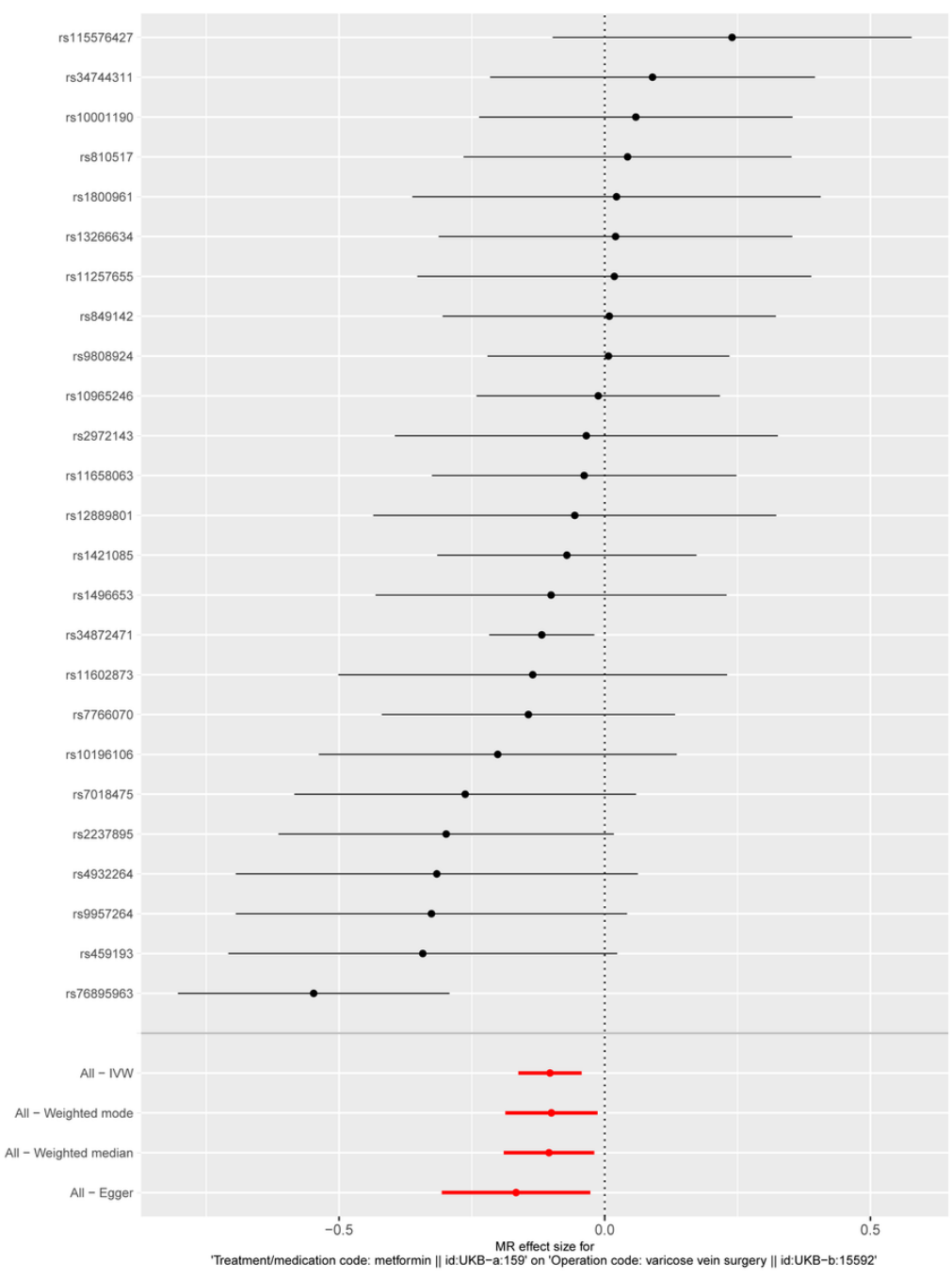

Figure 1 
Forest plot of single nucleotide polymorphisms (SNPs) associated with metformin and risk of varicose vein. Each black point represented the MR estimates for treatment of metformin on risk of varicose vein (rs10001190, rs10196106, rs10965246, rs11257655, rs115576427, rs11602873, rs11658063, rs12889801,

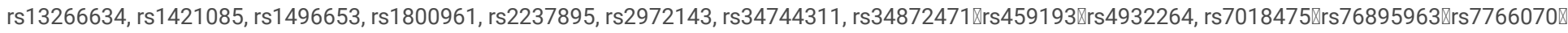
rs810517, rs849142, rs9808924, rs9957264). Red points showing the overall causal effect including all SNPs using four different methods (inverse-variance weighted [IVW] method, weighted mode, weighted median method and MR-Egger method). The horizontal lines represent $95 \%$ confidence intervals of the MR estimates.

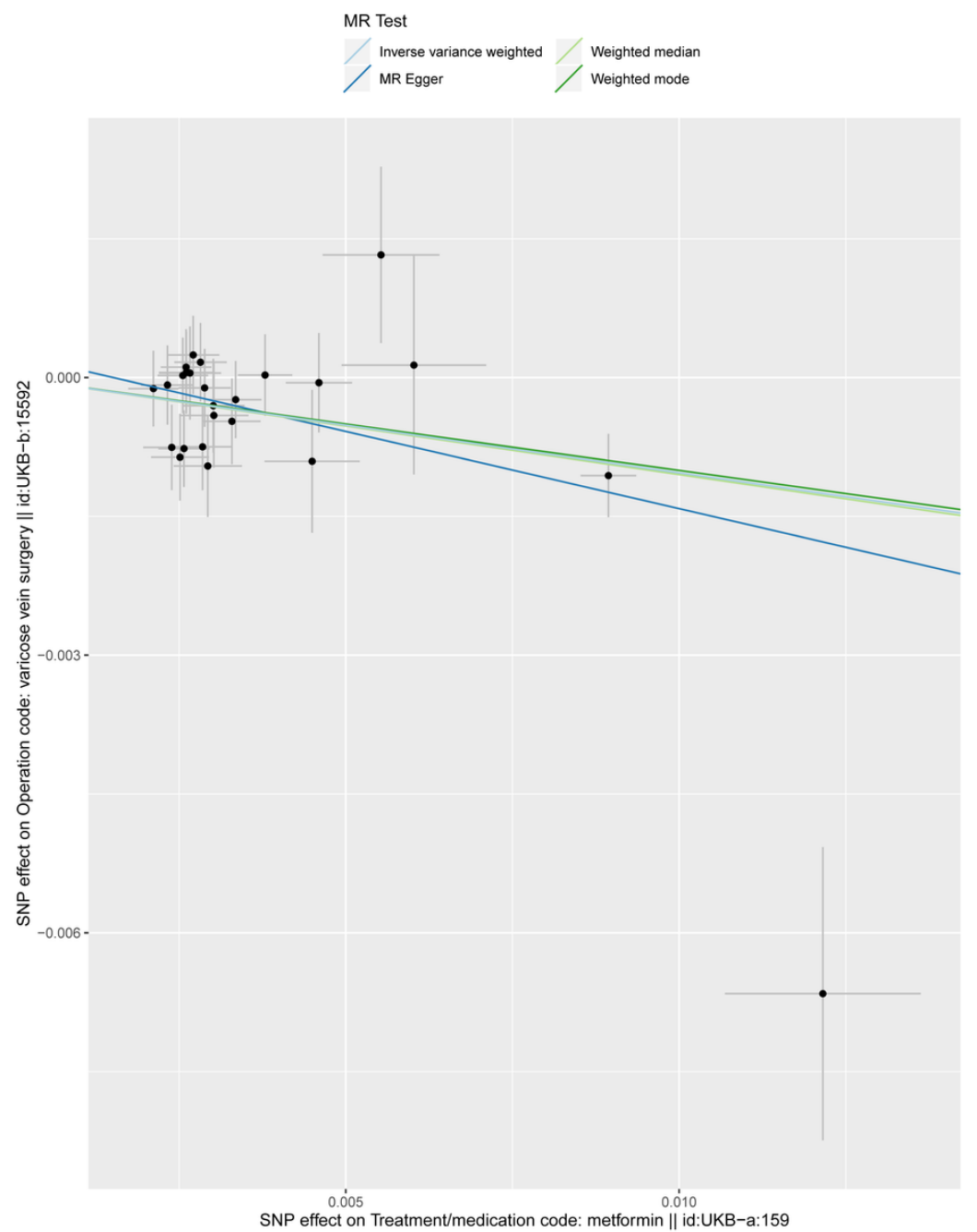

Figure 2

Scatter plot of SNPs associated with metformin and the risk of varicose vein. The plot relating the effect sizes of the SNP-metformin association (x-axis, SD) and the SNP-varicose vein associations ( $y$-axis, log (OR)) with $95 \%$ confidence intervals. The regression slopes of the lines correspond to causal estimates using four MR methods including the IVW approach, Weighted mode, Weighted median, and MR-Egger method. 


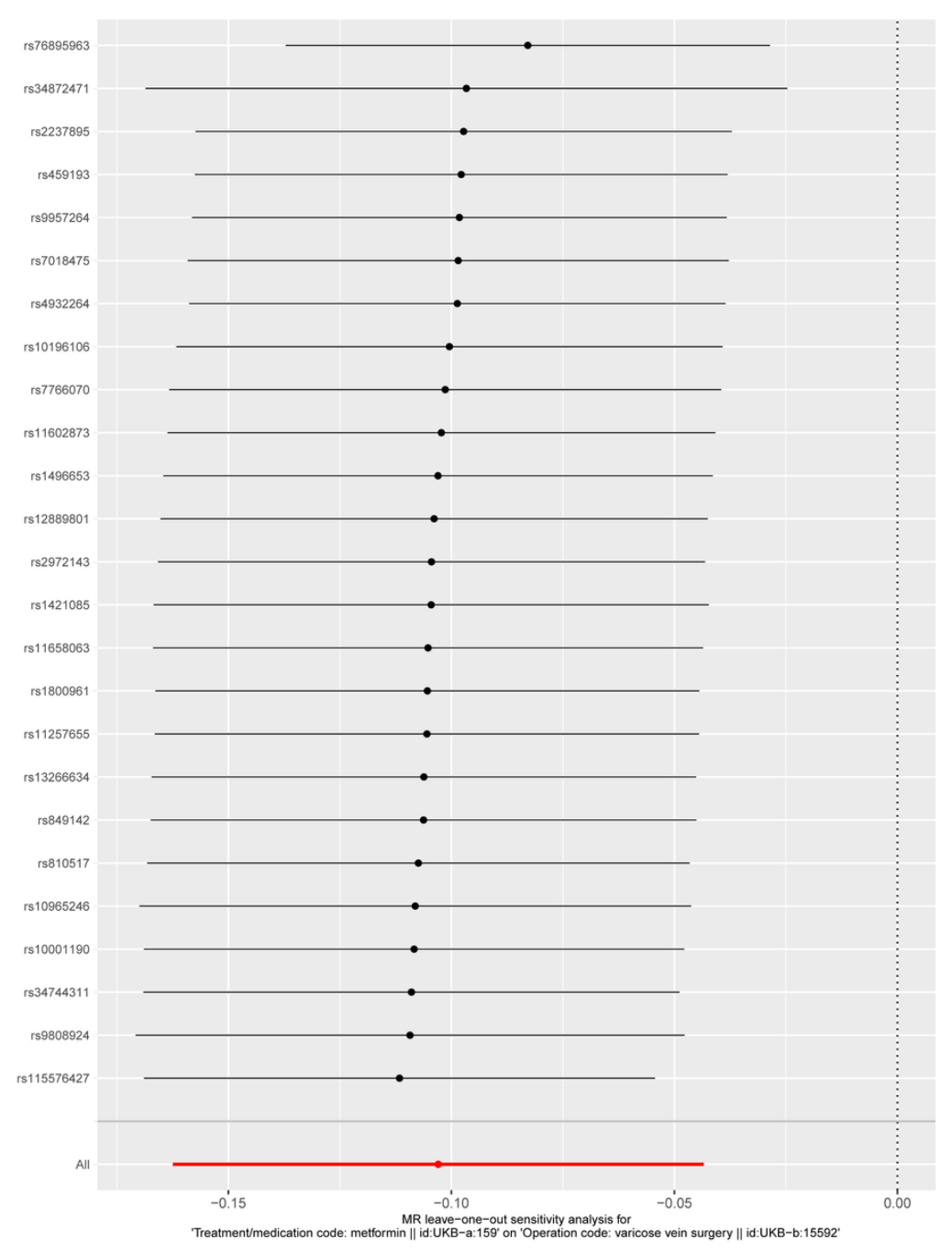

\section{Figure 3}

Leave-one-out (LOO) sensitivity analysis of SNPs associated with metformin and risk of varicose vein. Each black point represents the effect size( $\beta$ ) excluding one SNP estimated by the IVW method (rs10001190, rs10196106, rs10965246, rs11257655, rs115576427, rs11602873, rs11658063, rs12889801,

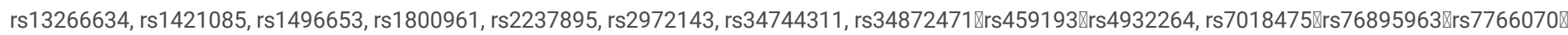
rs810517, rs849142, rs9808924, rs9957264, respectively). The red point depicts MR estimate including all SNPs. The line represents $95 \%$ confidence interval of MR estimates. According to the result of LOO analysis, no single SNP is strongly driving the overall effect of metformin on varicose vein.

\section{Supplementary Files}

This is a list of supplementary files associated with this preprint. Click to download.

- Supplementtable2.docx

- Supplementtable1.docx 